\title{
La interpretación asociativa del proceso creador
}

\section{Manuela Romo Santos}

\author{
Departamento de Psicología General \\ Universidad Autónoma de Madrid
}

\section{FUNDAMENTOS EXTRAPSICOLOGICOS DE LA HIPOTESIS}

"Un soir, je pris du café noir, contrairement a mon habitude, je ne pus m'endormir; les idées surgissaient en foule; je les sentais comme se heurter, jusqu'à ce que deux d'entre elles s'accrochassent pour ainsi dire pour former une combinaison stable."

Permítaseme el recurso de transcribir literalmente unas palabras de Henry Poincaré pronunciadas para describir una experiencia personal muy particular. Efectivamente, estas palabras servían para introducir, a un auditorio de psicólogos, en la descripción de las condiciones mentales que acompañaron en una noche de insomnio a la formulación básica de uno de sus más grandes descubrimientos, el que consagró su gloria: la teoría de los grupos fuchsianos y de las funciones fachsianas.

En el interesante libro de Hadamard: «Essai sur la psychologie de l'invention dans le domaine mathématique" (1945) se encuentra esta cita (pág. 24), así como el análisis en términos psicológicos del proceso de creación referido en la conferencia de Poincaré. Tal conferencia fue pronunciada ante la Société de Psychologie de París en 1908.

Queda bastante explícito con esta cita el carácter asociativo que un matemático reconocidamente creador confiere al proceso de creación.
En gran medida el fundamento de la hipótesis asociativa de la creatividad procede de campos ajenos a la psicología: de la matemática, de la física, de la literatura, de la música..., de aquellos campos donde pueden identificarse obras creadoras, obras que trascienden ese ámbito científico o artístico donde surgieron y que de forma más o menos universal son definidas como tales; de aquellos campos y de aquellas personas responsables de estas obras.

En la época en que la psicología de la creatividad estaba durmiendo el sueño de la giftedness ${ }^{1}$, los pocos intentos de definición del proceso eran muchas veces extrapsicológicos, como el libro de Hadamard o el análisis del mismo Poincaré, y cuando los hacía algún psicólogo nos encontramos con una gran profusión de citas introspectivas de artistas o científicos que fundamentan las deducciones sobre tal proceso psicológico, tal como sucede en el libro de Ghiselin (1952) y no tanto en la obra de Patrick, donde ya se da un tratamiento experimental al intento de definir las fases del proceso (Patrick, 1937, 1938 y 1941).

Pues bien, estos intentos de definición del proceso describían la fase de incubación como una actividad combinatoria, no consciente, que terminaba en el descubrimiento de esa "feliz idea» que no era sino la combinación más fecunda de las posibles y donde el azar juega un papel más o menos determinante. Dice Hadamard: 


\section{Estudios}

"De hecho, es evidente que la invención o el descubrimiento, ya se trate de matemáticas o de otraccosa, se produce por combinación de ideas. Existe un número extraordinariamente grande de tales combinaciones, la mayor parte desprovistas de interés, mientras que muy pocas de entre ellas pueden ser fecundas. ¿Cuáles son las que nuestro espíritu -quiero decir nuestro espíritu consciente- percibe? Unicamente aquellas que son fecundas $o$, excepcionalmente, aquellas que podrían llegar a serlo» 2 .

En esta primera parte introductoria cuya naturaleza es descriptivo-introspectiva vamos a ver algunas referencias más de interés acerca de las experiencias de genios reconocidos en las ciencias, las artes o las letras.

\section{Mozart:}

"Cuando estoy completamente conmigo mismo, enteramente solo y de buen humor, como ocurre, digamos, dando una vuelta en un carricoche o paseando a pie después de una buena comida o durante la noche cuando no puedo dormir, es en tales ocasiones cuando mis ideas fluyen mejor y más abundantemente. De dónde y cómo vienen, no lo sé; ni yo puedo forzarlas. Aquellas ideas que me agradan las.retengo en la memoria y estoy acostumbrado, según me han dicho, a tararearlas para mí mismo, en voz baja; si continúo de esta manera, pronto se me ocurre que puedo combinar este o aquel manjar para que encajen, de forma que haga con ellas un buen plato, es decir, de acuerdo con las reglas del contrapunto y con las peculiaridades de los diversos instrumentos, etc. (...). Mi tema va creciendo, se vuelve metódico y definido y el conjunto, aunque sea largo, se presenta ante mi mente casi completo y terminado, de manera que puedo examinarlo de una ojeada, como si se tratase de un hermoso cuadro o de una bella escultura» ${ }^{3}$.

O el 'genio de Galton (quién sabe si un análisis sobre sus propias reflexiones le hubiera llevado a una interpretación de la creatividad más abierta y comprensiva que su idea del «genio»):

"Cuando estoy meditando sobre alguna cosa, me parece que el proceso de esta meditación es el siguiente: las ideas que se encuentran en un momento dado en plena conciencia parecen sobresalir ellas mismas, las más apropiadas de entre un cierto número de otras ideas que se encuentran próximas. Todo pasa como si hubiera en mi espíritu una sala de audiencia donde la plena conciencia tiene un salón donde dos o tres ideas comparecen simultáneamente; y, a la vez, una antecámara llena de ideas más o menos aparentes, situada justo detrás de la vista de la plena conciencian".

\section{Einstein:}

«Las palabras o el lenguaje no parecen jugar ningún papel en mi mecanismo de pensamiento. Las entidades físicas que parecen servir como elementos en el pensamiento son ciertos signos e imágenes más o menos claros que pueden ser combinados (...). Este juego combinatorio parece ser el hecho esencial en el pensamiento productivo"s.

También los Mednick nos citan las palabras de André Breton describiendo un collage de Ernest en los siguientes términos:

«... una maravillosa capacidad para abarcar dos realidades mutuamente distantes sin ir más allá del campo de nuestra experiencia ${ }^{6}$.

Y qué decir del espectacular sueño de $\mathrm{Ke}$ kulé, origen, según parece, del descubrimiento de la estructura de la molécula del benceno. Puede admitirse que eran ciertamente remotas las dos realidades combinadas aquí para dar lugar al descubrimiento químico:

"Volvi mi silla hacia el fuego y dormité. De nuevo los átomos estaban brincando ante mis ojos. Los grupos más pequeños se mantenían molestamente al fondo. Mi ojo mental, agudizado por visiones de esta clase, podía ahora distinguir enormes estructuras replegadas; largas filas, algunas veces muy fuertemente unidas, emparejadas y recogidas en una serpenteante imagen. Pero ¿qué era aquello? Una de las serpientes se había agarrado fuertemente de su propia cola y la forma giraba rápidamente burlándose ante mis ojos. Me desperté como por un relámpago" 7 .

Como vemos en las últimas citas la le janía de las asociaciones es determinante en la creatividad del producto. Tal es precisamente la «remotedness», una dimensión vertebradora de la teoría de Mednick y encuentra su más grande sustento en las formulaciones de Poincaré que se identifican, como veremos, con la defínición que los Mednick van a dar del pensamiento creador en el año 1962. Para Pincaré:

«La creación consiste en hacer nuevas combinaciones de elementos asociativos que son útiles. En el campo matemático los hechos más disgnos de ser estudiados se revelan como aquellos que consisten en insospechadas relaciones entre otros bien conocidos, pero fuertemente considerados como extraños el uno al otro. Entre las combinaciones elegidas, la más fértil a menudo se 
formará de elementos procedentes de dominios muy lejanos" ${ }^{8}$.

La concepción asociativa de la creatividad estaba en el ambiente. No es entonces casualidad que en el año 1962 un profesor de la Universidad de Michigan, Sarnoff A. Mednick publique un trabajo titulado «The Associative Basis of the Creative Process", donde se desarrolla una teoría asociativa sobre el pensamiento creador. No es casualidad, ni tampoco se trata de la única formulación de la creatividad en términos asociativos, aunque sí la más elaborada, la que ha buscado una confirmación en la práctica del modelo cual ha sido el Test de Asociaciones Remotas a la vez que una dimensión aplicada y la que ha dado lugar a más trabajos posteriores, bien a favor, bien en contra, y que voy a hacer objeto de análisis en este artículo.

\section{CONCEPCIONES ASOCIATIVAS DE LA CREATIVIDAD HASTA EL AÑO 1962}

Este caldo de cultivo que hizo brotar con gran empuje un modelo asociativo del pensamiento creador no sólo se alimentaba de las experiencias vitales contadas por algunos genios. Veamos desde dentro de la psicología las aproximaciones que se fueron haciendo hasta el año 1962 a la hipótesis asociativa:

Ghiselin, en su obra The Creative Process (1952), pone claramente de relieve que un hecho común en la vida de los genios, a los que él cita, es que el proceso creador parece ser esencialmente un fenómeno de sintetizar asociaciones, $\mathrm{y}$ añade que la condición más necesaria de la creatividad es que se presente una configuración nueva, una nueva constelación de datos con una significación sin precedentes específicos.

El precedente más aproximado a la teoría asociativa de la creatividad definida por Mednick, es el de Maltzman en sus estudios sobre originalidad, concepto que define así:

*... conducta relativamente infrecuente, no es común bajo condiciones dadas, pero es relevante a esas condiciones" ${ }^{9}$.

Utiliza el concepto de "gradiente asociativo» para referirse a las diferencias individuales en cuanto a originalidad. La singularidad de las respuestas en tareas asociativas se puede conceptualizar en términos de «llanura» (flatness) del gradiente asociativo de las personas.
Maltzman, en su concepción experimentalista, ha llevado a cabo numerosos trabajos que evidencian la transferencia del entrenamiento en tareas asociativas. El método consiste en entrenar al sujeto para que ofreciera en sus respuestas una diversidad de posibilidades asociativas, lo cual favorece la probabilidad (ésta es la hipótesis) de que las mismas sean más remotas o únicas.

El procedimiento estándar consiste en presentar repetidas veces una misma palabra estímulo a los sujetos para que respondan con otra diferente en cada presentación. Esto obliga a buscar cada vez más respuestas originales. El objetivo planteado en la hipótesis es comprobar que la ocurrencia de respuestas originales facilita la probabilidad de ocurrencia de otras respuestas originales por un proceso de generalización.

En un experimento (Maltzman y cols., 1960), se determinó el efecto de transferencia de una situación experimental consistente en responder con una palabra diferente cada vez a otra que era repetida cinco veces. La lista contenía veinticinco palabras diferentes, siendo la variable dependiente la ejecución en una tarea de «usos». L.os resultados fueron éstos: los usos menos corrientes para un objeto dado se ofrecían cuando previamente el sujeto ha sido entrenado en una tarea para asociar elementos poco probables a palabras dadas. La "altura" o "llanura" del gradiente asociativo, concluye Maltzman, es un concepto pertinente para interpretar los resultados de un test de usos.

En este sentido se explica que las personas que han sido entrenadas para producir gradientes asociativos planos muestren indecisión ante una tarea consistente en ofrecer una única respuesta asociativa a una palabra dada. Se interpreta esto como un conflicro entre respuestas alternativas. Así explica Maltzman la falta de resultados cuando al entrenamiento siguen tareas asociativas que requieren asociaciones inusuales, pero determinadas por el experimentador. Cuanto más estricto y específico es el criterio más negativos son los resultados.

En este punto se halla la diferencia con Mednick. Maltzman y asociados (1964) aportan los datos de un experimento donde comprueban que las puntuaciones altas y bajas en el RAT no discriminan entre jerarquías asociativas, poniendo en tela de juicio la validez de constructo de este test.

Entre las interpretaciones cognitivistas, la definición de creatividad de Neisser (1963) tiene que ver con un proceso múltiple, un con- 
junto de operaciones donde el pensamiento discursivo interactúa con los elementos asociativos inconscientes.

Por su parte, Bruner, en el año 62, define la creatividad como una sorpresa eficiente, resultado de una actividad combinatoria donde las cosas se clasifican en una nueva perspectiva. Tal eficiencia la explica de tres formas, aunque éstas se encuentran presentes simultáneamente en todos los actos creativos. La eficiencia formal encuentra su terreno más fértil en el campo de las matemáticas:

«Este fenómeno consiste en la organización de los elementos de tal forma que se perciben relaciones que no eran evidentes antes, reagrupaciones que no existian, formas de reunir los elementos que antes no eran posibles. La homogeneidad, la armonía y la profundidad de las relaciones son los resultados a los que se llegas ${ }^{10}$.

Y se apoya en la concepción de Poincaré, donde se habla de combinaciones consistentes en establecer parentescos insospechados entre hechos conocidos desde hace mucho tiempo, pero concebidos erróneamente como extraños unos a otros.

La eficiencia metafórica también soporta una interpretación asociativa, pues enlaza partes diferentes de la experiencia, siendo el vínculo de unión el arte. Tales lazos prescinden de una clasificación sistemática, se trata de relaciones insospechadas anteriormente. Clarificadoras al máximo son las siguientes palabras de Picasso:

"Qué triste es para un pintor al que le gustan las rubias tener que prescindir del placer de ponerlas en su cuadro porque no van bien con la cesta de frutas. Qué desgracia para un pintor que detesta las manzanas tener que utilizarlas todo el tiempo porque armonizan con el mantel. Yo introduzco en mi cuadro todo lo que me gusta. Mala suerte para las cosas, no tienen más que arreglarse entre ellas" 11 .

Veamos ahora otras interpretaciones del proceso que no tratan la dimensión cognitiva $y$, no obstante, hablan también del proceso asociativo.

Para Rogers la actividad combinatoria requerida para la creatividad tiene un sentido lúdico en cuanto se identifica con la actividad infantil típica que tiene un carácter oscilante y escasamente racional. Tales caracteristicas son propias de los procesos primarios, cuya importancia es reconocida en la creatividad. Estos proporcionan al pensamiento un sentido lú- dico donde el azar tiene importancia en la aparición de combinaciones creadoras que no tienen cabida en el pensamiento discursivo racional.

Entre las interpretaciones sobre la creatividad de la psicología profunda la de Cubie (1958) es también asociativa: Esa «regresión al servicio del yo" que es la creatividad consiste en el descubrimiento de nexos inesperados entre las cosas, producto de la libre asociación de los procesos simbólicos preconscientes. En Júng (1959) es la integración y resolución de dicotomías y la fusión de procesos primarios y secundarios.

Y si hablamos de los correlatos motivacionales y perceptuales de la creatividad podríamos encuadrar fácilmente en el contexto que estamos tratando la tan cacareada «tolerancia a la ambigüedad» de la personalidad creadora donde se muestra la particularísima habilidad de asumir simultáneamente e integrar los contrarios. Y en la interpretación de Barron sobre las preferencias perceptivas hacia la complejidad asimétrica también hay evidencia de una búsqueda de integración:

«El individuo creador es aquel que tiene una necesidad especialmente fuerte de encontrar orden donde en ningún modo aparece» ${ }^{12}$.

Finalmente, es obligado decir algunas palabras sobre Guilford. A pesar del aparente antagonismo entre los enfoques factorial y asociativo en el estudio de la creatividad conviene, sin embargo, dejar claras un par de cuestiones. Me refiero a la definición de dos de los factores cuya relevancia en la explicación del pensamiento creador ha quedado más clara desde el principio: los factores de fluidez asociativa y de originalidad.

En el primer caso se define aquella forma de productividad semántica capaz de enlazar significados valorándose con pruebas asociativas, como el test de "Associational Fluency" donde se trata de escribir diferentes sinónimos para una palabra dada.

En cuanto al factor de originalidad, encontramos en Guilford una dimensión operativa del mismo basada en índices de distancia asociativa de factura casi idéntica a la del test de Asociaciones Remotas (RAT) de Mednick donde se evalúa la capacidad para encontrar las conexiones menos obvias, más tenues que existen entre las cosas, a base de buscar en cada ítem del test una palabra que se relaciona simultáneamente con otras dos ya dadas. 


\section{LA TEORIA ASOCIATIVA DE MEDNICK}

\section{III.1. Significación científica de la teoría}

Siguiendo con las argumentaciones con respecto a la teoría que se va a definir hay que señalar una cuestión interesante que califica favorablemente la teoria de Mednick.

Como han señalado Rothemberg y Hausman (1976), Mednick es uno de los pocos psicólogos que empieza la investigación en creatividad con una teoría definida del proceso creador y continúa con la investigación empírica. Tal cosa no podemos decirla de muchos otros autores en este campo.

En gran cantidad de ocasiones se ha insistido sobre la necesidad de disponer de una teoría para acometer con paso firme una investigación. Pero, sobre todo, el problema ha sido tratado en la polémica relativamente reciente sobre la metodología experimental versus la diferencial que ha puesto de relieve el problema planteado en la psicología de la inteligencia (problema que no es ajeno a lo que estamos tratando) y otros campos. La obra de Eysenck y los famosos trabajos de Cronbach $(1957,1975)$ parecen haber dado una salida a esta encrucijada. Eysenck (1966) ha puntualizado (y su obra es buen ejemplo de ello) la necesidad de tener en cuenta la comprensión y definición de los procesos tanto como el estudio de las diferencias individuales.

Pues bien, Mednick es otro ejemplo de lo mismo. Mednick no ha esperado a construir su test para dar una definición operativa del pensamiento creador. El test se ha construido para verificar ciertas hipótesis elaboradas a partir de una teoría o modelo previo.

En lo dicho va implícito un argumento de validez que posee esta teoría asociativa; se trata de una aproximación experimental a la creatividad, aunque, de acuerdo con Rieben (1979), los estudios experimentales en este campo tienen un valor limitado, ya que, aunque se centre en el estudio de los procesos, las más de las veces los argumentos para definirlos son intuitivos e introspectivos; como desgraciadamente sucede en gran medida en el caso de Mednick.

Veamos ya la definición de pensamiento creador de Mednick:

«La formación de elementos asociativos en nuevas combinaciones que cumplen reque- rimientos específicos o son en alguna manera útiles. Cuanto más mutuamente remotos sean los elementos de la nueva combinación más creativo es el proceso de solución» ${ }^{13}$.

En términos asociativos Mednick concibe tres formas de alcanzar una solución. Tres formas de relacionarse la información para dar lugar a esa combinación creadora:

1. Por contigüidad. En el campo científico se han producido descubrimientos de esta forma que ha dado en llamarse «serendipity" ${ }^{14}$. Para hacernos una idea de en qué consiste este fenómeno que podríamos interpretar de «feliz casualidad», veamos un ejemplo típico citado por Simon que fue el descubrimiento de la vulcanización del caucho:

«... cuando Goodyear dejó caer accidentalmente unas gotas de dicho material sobre una estufa caliente y pudo observar cómo el caucho resultante tenía las propiedades que él precisamente iba buscando (...). Esta observación fortuita de un fenómeno nuevo (serendipity) suele acontecer casi siempre a una mente preparada para ello y no precisamente a cualquiera que haya pensado un cuarto de hora sobre determinado problema" 15

Por su parte, Mednick cita la divertida anécdota de aquel científico que hizo de la serendipity su método de investigación: colocaba en un recipiente papelitos conteniendo cada uno escrito un hecho físico, de vez en cuando revolvía los papeles y sacaba dos al azar, buscando algún descubrimiento basado en nuevas $\mathrm{y}$ útiles combinaciones.

2. Por similaridad. Esta combinación asociativa es importante en aquellos ámbitos que dependen poco de la manipulación de símbolos, como son la creatividad artística y literaria.

3. Por mediación de elementos comunes. Importante en aquellas áreas donde el uso de símbolos es determinante.

Siguiendo con las diferencias dentro del proceso, cabe distinguir también entre aproximación perceptual y conceptual a los problemas como dos formas de alcanzar soluciones creadoras, bien que la combinación de asociados se haga sobre representaciones concretas de aspectos relevantes del problema, bien que requiera utilizar información conceptual.

Finalmente otro criterio diferenciador se refiere a la dimensión visualizadora-verbalizadora que da origen a diferencias individuales en relación con la facilidad en el tratamiento de la información. 
Aspectos como estos citados pueden ser parcialmente responsables, según Mednick, de las aptitudes diferenciales para el trabajo creador en diferentes campos.

Pasemos ahora con cierto detenimiento a analizar los determinantes del proceso.

\section{III.2. Conceptos de fuerza y jerarquía asociativas como ejes de la teoría}

¿De qué depende la presencia de soluciones creadoras a los problemas? ¿Cómo se llega a esa combinación asociativa que cumple los requisitos establecidos en la definición?

La clave está en los conceptos de fuerza y jerarquía asnciativas. La organización de las asociaciones en el individuo determina la probabilidad y rapidez en alcanzar una solución creadora. La persona capaz de hallar mayor número de asociaciones y menos comunes entre los conceptos será la más capacitada para llevar su pensamiento por los caminos de la creatividad. El concepto de jerarquía asociativa operativiza esta afirmación: para un estímulo dado, la cantidad de asociaciones posibles que es capaz de producir un sujeto sería su jerarquía asociativa. Existe una correlación negativa entre el número de asociaciones y la fuerza de asociación.

Sobre estos presupuestos, Mednick distingue entre jerarquías asociativas escarpadas y planas:

\section{TABLA 1}

Jerarquias asociativas hipotecarias para la palabra estimulo "mesa" (Tomado de Mednick, S. A. 1962)

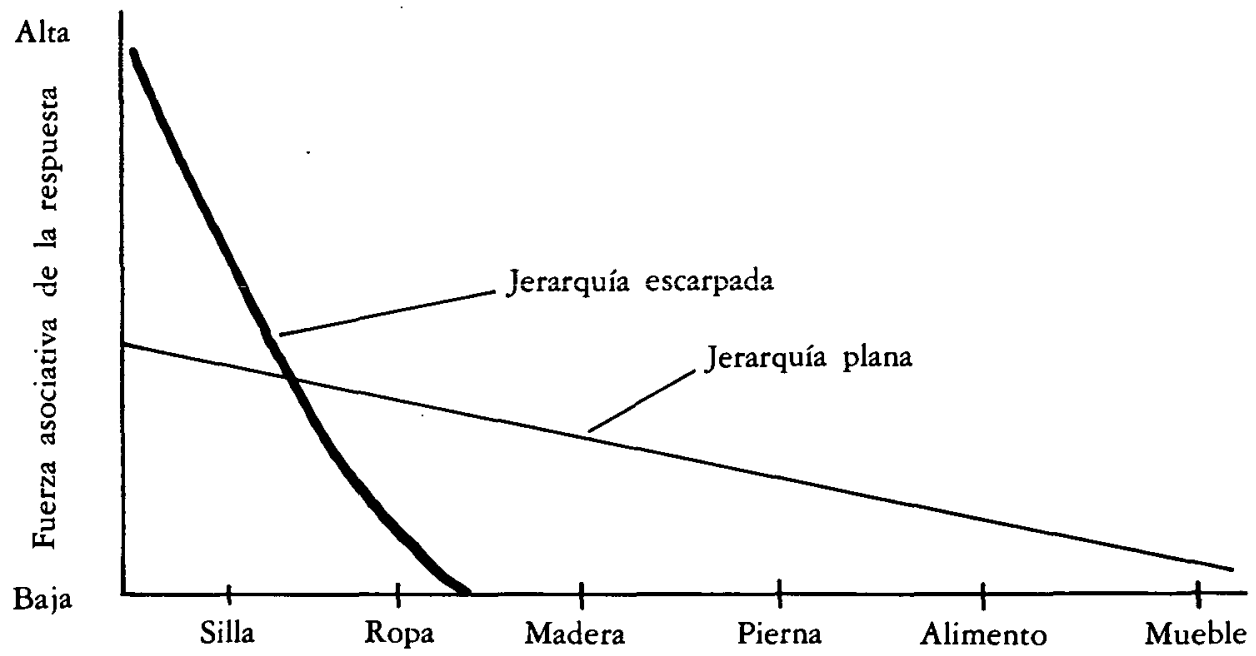

Un gradiente asociativo plano supone una distribución generosa de la fuerza asociativa entre muchas ideas, no hay una única asociación dominante con el estímulo, y la probabilidad de encontrar asociaciones remotas aumenta. Entre aquellas respuestas más remotas se encuentran los elementos mediadores requeridos para una solución creadora.

Por el contrario, con una gran concentración de fuerza asociativa en un número reducido de respuestas estereotipadas la probabilidad de encontrar soluciones creadoras es mínima. Tal sucede en un gradiente asociativo escarpado.
Es preciso hacer una salvedad, no obstante, $y$ es el caso de algunos individuos creadores con gradiente asociativo escarpado, pero a la vez desviado, es decir, donde la fuerza asociativa se concentra en un número reducido de asociaciones que no son estereotipadas, sino diferentes de las asociaciones vulgares dominantes. Este fenómeno, según apunta Mednick, es relativamente común entre algunos escritores; tal es el caso del novelista o el cineasta de «una sola obra» cuyos trabajos parecen remitirse siempre a aquel primer éxito.

Pero este no es un caso representativo de 


\section{Estudios}

lo que entendemos por personalidad creadora, donde tanto la productividad como la originalidad son una constante. $Y$ es en estas personas donde toman forma los principios enunciados.

Cuanto más grande sea la capacidad de establecer asociaciones entre los conceptos de un problema mayor será la probabilidad de hallar una solución creadora. Los individuos con gran fuerza asociativa en pocas respuestas no proliferan en sus asociaciones. Cuantas más asociaciones evoque un elemento del problema, mayor probabilidad de establecer puentes de mediación con otro elemento requerido para encontrar una combinación creadora.

Tanto la productividad como la unicidad («uniqueness») de las asociaciones son las dimensiones relevantes para dar con esa combinación creadora.

Veamos alguna confirmación experimental, aunque la literatura sobre el tema presenta resultados claramente contradictorios. Tal confusionismo se difumina en gran parte si no tomamos como criterio discriminador el Test de Asociaciones Remotas. Quiero decir que cuando se ha tomado como variable dependiente este test no siempre se ha podido confirmar que un aumento de la productividad o la originalidad mejora la ejecución de la prueba, pero esto es un problema relacionado con la validez del test que en seguida se tratará; y ahora, para no introducir confusionismo, veremos algún ejemplo cuya variable dependiente no sea el Test de Asociaciones Remotas.

Así Mednick (1962) cita una investigación suya con un grupo de científicos diferenciados por su creatividad investigadora. Los cientificos no creadores dieron en un 80 por 100 más respuestas estereotipadas al KentRosanoff que los creadores. Esto autoriza a decir que la conducta verbal asociativa de los individuos altamente creadores se caracteriza por menor estereotipia y comunalidad.

Precisamente los experimentos de Maltzman sobre la transferencia del entrenamiento en la unicidad asociativa son a la vez un fuerte apoyo a lo que estamos diciendo. Es decir, que el aumento de la productividad en tareas asociativas determina un incremento de la probabilidad de ofrecer respuestas originales.

Ahora bien, no debe pensarse que la selección de las asociaciones más extrañas entre sí más remotas, sea un resultado casual en de- pendencia de esta productividad. En un experimento de Houston y Mednick (1963) donde se seleccionaron los grupos extremos en cuanto a creatividad, el grupo superior mostraba preferencias hacia términos que implicaban nuevas asociaciones verbales en una tarea de elección de palabras. Lo contrario sucedía con el grupo bajo en creatividad. De esto concluyen que la preferencia hacia los asociados remotos no es casualidad, sino. un fin en sí mismo.

El pensador creativo ante una tarea rechazaría las asociaciones comunes y buscaría las que sean distantes, inaccesibles.

La interpretación de todos estos resultados dentro de la teoría que estamos viendo tiene que ver con la definición asociativa del fenómeno de la incubación.

Así lo exponen los Mednick en las conclusiones de un experimento sobre entrenamiento en una tarea asociativa (Mednick, $M$ T., Mednick, S. A. y Mednick, E.U., 1964):

Tras la aplicación a los sujetos de un test de asociados remotos, éstos tenían que realizar una tarea asociativa consistente en resolver analogías con los términos específicos de algunos de los problemas no resueltos. Este entrenamiento produjo un descubrimiento de las soluciones no sólo en los items entrenados, sino en otros no entrenados y no resueltos. Por otra parte, los individuos más creadores aprovecharon mejor el entrenamiento que los no creadores.

Concluyen que el fenómeno de la incubación supone un cambio en las probabilidades de asociar los elementos del problema para llegar a una producción creadora y se apoyan en las definiciones que de esta fase del proceso han hecho los estudiosos del mismo entendiéndolo como un proceso asociativo inconsciente. Incluso Hull habla de un momento en la solución de problemas donde el pensador debe hacer intentos para combinar porciones de hábitos nunca asociados anteriormente, hasta que la solución más oportuna se hace consciente (Hull, 1953).

Sobre lo dicho resulta oportuno pasar a plantear una cuestión de importancia: ¿Cómo llega la combinación creadora? ¿Por qué es elegida esa combinación de entre todas las posibles?

Para responder estas preguntas podríamos, replicando a Ghiselin, sumergirnos en mil citas de otros tantos personajes contando sus experiencias de iluminación. Contar anécdotas so- 


\section{Estudios}

bre si fue al afeitarse, al tomarse unas cervezas, al subirse al autobús... y sobre los estados afectivos asociados al hecho. Pero más que la impresión emocional del «jajá!» nos interesa el contexto intelectual que justifica esa satisfacción de haber dado con lo que buscábamos y en el contexto de Mednick que nos ocupa, es así como se llega a la solución:

«La explicación del proceso de selección está relacionada con la naturaleza del problema. Los problemas ocasionan un especifico y relativamente objetivo set de criterios testables" ${ }^{16}$.

$Y$ sigue diciendo que estos criterios imponen una selección entre los estímulos a elegir para entrar en combinación, así como la valoración de las combinaciones resultantes.

Alguna relación con esto tiene lo que decía Renoir sobre sus cuadros: cuando se sentía en la tentación de pellizcar la nalga de la mujer que pintaba consideraba acabada su obra.

Otra dimensión del problema nos lleva a plantear esta pregunta: ¿Cómo determinar el grado en que una asociación remota es creadora?

Mednick intenta curarse en salud desde el principio, salvando el principio de «pertenencia»: el producto es pertinente en la situación que le dio origen; y de este modo quiere ahorrarse las críticas recibidas por Maltzman y su «conducta original» y así distingue pensamiento creador de pensamiento original en base a los requerimientos de utilidad. El pensamiento original, por el contrario, es algo que prolifera en instituciones psiquiátricas.

No obstante la polémica se ha planteado, no tanto sobre su definición de pensamiento creador cuanto por el instrumento que pretende medirlo: el Test de Asociaciones Remotas. Así Hood (1969), en un trabajo rechaza el test como medida de algo más que ejecución original, dado que existen dificultades conceptuales en la definición del pensa- miento creador de Mednick que indican que la distinción entre originalidad y creatividad es arbitraria.

Por su parte, en la réplica, Mednick se defiende recalcando que la solución creadora implica algo más que originalidad porque debe cumplir unos requerimientos o criterios específicos; y añade que la creatividad de la solución debe ser evaluada en función del número de requerimientos que cumple el producto.

Subyace a esta polémica el problema ya pergeñado de la falta de identificación entre la teoría y el instrumento.

La más directa aplicación de este principio enunciado acerca de que la creatividad del producto es función del número de requerimientos específicos que cumple, está - según Mednick - en el laboratorio, donde las tareas, requerimientos y soluciones pueden ser arbitrariamente construidas y variadas.

En este momento entramos ya en el terreno más resbaladizo de la formulación de Mednick: es, precisamente, el momento de entrar en el laboratorio de investigación y construir un test donde la combinación específica de los criterios precisos que debe encontrar el sujeto está predeterminada por el experimentador.

La impresión del escepticismo que se siente al llegar a este punto de la teoría de Mednick es general, aunque llega a diluirse cuando uno analiza los orígenes del Test de Asociaciones Remotas. Pero es inevitable una reflexión en estos términos: parece algo contrario al espíritu de la creatividad plantear tareas donde lo que se va a crear esté ya previamente descubierto. Tal es el caso del Test de Asociaciones Remotas: un test de respuesta única, prefijada de antemano y que pretende medir la creatividad. Pero el análisis y la crítica del RAT es otro tema aparte que rebasa los límites de este trabajo de revisión teórica y que, por su extensión, merece tratamiento aparte.

\section{Notas}

1 Me refiero al segundo período histórico que, dentro de la clasificación de Getzels (1975), define una etapa muy diferenciada en la investigación de la creatividad; la que viene marcada por la hegemonía del $\mathrm{CI}$ como exclusivo método de evaluación de las capacidades intelectuales y, en consecuencia, la consideración de las formas de pensamiento creador como asociadas al genio, a un CI excepcionalmente elevado (giftedness).

2 J. Hadamard: Essay sur la Psychologie de linvention dans le Domaine Matématique, 1975, pág. 36.

3 W. A. Mozart: A. Letter, en P. E. Vernon: Creativity: Selected Readings, 1973, pág. 174. 
4 F. Galton: Inquiries into Human Faculty, 1908, pág. 146.

5 A. Einstein. Citado en S. A. Mednick: Remote Associated Test. Examiner's Manual, 1976, pág. 1.

6 S. A. Mednick, op. cit., pág. 1. pág. 83.

7 F. A. Kekulé. Citado en J. C. Gowan: Some New Thoughts on the Development of Creativity, 1978,

8 En S. A. Mednick, op. cit., pág. 1.

9 I. Maltman y cols.: Experimental Studies in the Training of Originality, 1960, pág. 1.

10 J. Bruner: Condiciones de la Creatividad en A. Beaudot. La Creatividad, 1980, pág. 21.

11 P. R. Picasso. Citado en J. Bruner, op. cit., pág. 191.

12 Taylor-Barron: Scientific Creativity: its Recognition and Development, 1963, pág. 387.

13 S. A. Mednick: The Associative Basis of the Creative Process. En Rothemberg-Hausman, $T$ be Creativity Question, 1976, pág. 228.

14 No existe traducción en español con el significado que se le aplica al término en este contexto.

is H. A. Simon: La Comprensión de la Creatividad. En Gowan y cols.: Implicaciones Educativas de la Creatividad, 1976, pág. 55.

16 S. A. Mednick. En Khotemberg-Hausman, op. cit., pág. 233.

\section{Referencias}

En aquellos casos en que no se ha utilizado la edición original, el año de ésta aparece entre paréntesis junto al título de la obra.

Barron, F.: Personalidad Creadora y Proceso Creador (1969). Madrid. Marova, 1976.

BRUNER, J. S.: Condiciones de la Creatividad (1962). En Beaudot: La Creatividad.

Christensen, P. R., y Guilford, J. P.: "An Experimental Study of Verbal Fluency Factors». Brittish Journal of Statistical Psychology, 1963, 16, 1-26.

Cronbach, L. J.: «The two Disciplines of Scientific Psychology». American Psychologist, 1957, 12, 671-684.

Cronbach, L. J.: "Beyond the two Disciplines in Scientific Psychology». American Psychologist, febrero $1975,116-26$.

EINSTEIN, A., e INFELD, L.: The Evolution of Physics. Nueva York. Simon and Schuster, 1938.

EYSENCK, H. J.: «Personality and Experimental Psychology». Brittish Bulletin of Psychological Society, 1966, $19,1-28$.

GaLtoN, F.: Hereditary Genious: An Inquiry into its Laws and Consequences. Londres: MacMillan \& Company, 1869.

Griselin, B.: The Creative Process. Londres: Cambridge University Press, 1952.

GowaN, J.C.: "Some New Thoughts on the Development of Creativity". Journal of Creative Behavior, $1978,2,77-91$.

GowAN, J. C.; Demos, E., y TORRANCE, E. P.: Implicaciones educativas de la creatividad (1967). Salamanca, Anaya, 1976.

GuILFORD, J. P.: «La Creatividad: Retrospectiva y Prospectiva (1970)». En A. Beaudot: La Creatividad.

GUILFORD, J. P., y HOEPFNER, R.: The Analysis of Intelligence. Nueva York: McGraw Hill, 1971.

Hadamard, J.: Essai sur la Psychologi de l'invention dans le domaine mathematique (1945). París, Bordas, 1975.

Hood, R. W.: «On Creativity as Defined by the Remote Associates Test». Psychological Reports, 1969, 24 (3), 914.

Houston, J. P.; y MEDNICK, S. A.: "Creativity and the Need for Novelty». Joumal of Abnormal and Social Psychology, 1963, 66, 137-41.

HuLl, C. L.: "The Mechanism of the Assembly of Behavior Segments in Novel Combinations Suitable for Problem Solution». Psychological Review, 1953, 42, 219-45.

Kubuie, L. S.: Neurotic Distortion of the Creative Process. University of Kansas Press. Lawrence, 1958.

Maltzman, 1.: "On the .Training of Originality». Psychological Review, 1960, 67, 229-42.

Maltzman, 1., y cols.: «Experimental Studies in the Training of Originality. Psychological Monographs, $1960,74$.

Maltzman y cols.: «Experimental Studies of Associative Variables in Originality». Psycbological Monographs, 1964, 3.

Mednick, S. A.: "The Associative Basis of the Creative Process (1962)». En Rothemberg y Hausman: The Creativity Question.

MEDNIK, S. A.: "The Remote Associates Test». Journal of Creative Behavior, 1968, 2, 213-14.

MEDNICK, S. A.: «Reply to Hood's On Creativity as Defined by the Remote Associates Test». Psychological Reports, 1969, 25 (1), 194.

Mednick, S. A., y Mednick, M. T.: Remote Associates Test. Examiners Manual. Boston. Hught Mifflin, 1967. 


\section{Estudios}

Mednick, M. T.; Mednick, S. A., y MedniCK, E. U.: «Incubation of Creative Performance and Specific

Associative Priming». Journal of Abnormal and Social Psychology, 1964, 69, 84-88.

MOZART, W. A.: "A Letter» (¿1978?). En Vernon. Creativity: Selected Readings.

Neisser, U.: "The Multiplicity of Thought". British Journal of Psychology, 1963, 54, 1-14.

PATRICK, C.: "Creative Thought in Artist». Journal of Psychology, 1937, 4, 35-73.

Patrick, C.: “Scientific Thought". Journal of Psychology, 1938, 5, 55-83.

PATRICK, C.: “Whole and Part Relationship in Creative Thought». American Journal of Psycbology, 1941, 54, 128-131.

Poincare, H.: The Foundations of Science. Nueva York, Science Press, 1913.

ROGERS, C. R.: "Toward a Theory of Creativity». (1959). En Anderson: Creativity and its Cultivation, 1959.

Romo, M.: Dimensiones Cognitivas de la Creatividad. Tesis doctoral. Universidad Autónoma de Madrid, 1980.

Rothemberg, A., y Hausman, C. R.: The Creativity Question. Durham: Duke University Press, 1976.

SimON, H. A.: «La Comprensión de la Creatividad» (1964). En Gowan y cols: Implicaciones Educativas de la Creatividad.

TAYLOR, C. W., y Barron, F.: Scientific Creativity: its Recognition. Nueva York: Wiley and Sons, 1963.

TAYLOR, I. A., y Getzels, J. W.: Perspectives in Creativity. Chicago: Aldine, 1975.

WiLson, R. C.; Guilford, J. P., y CHRISTENSEN, P. R.: «The Measurement of Individual Differences in Originality». Psychological Bulletin, 1953, 50, 362-70.

\section{Resumen}

Este trabajo es una revisión de las aportaciones a la psicología de la creatividad, que proceden de las interpretaciones asociativas del proceso creador.

Las referencias introspectivas de los individuos excepcionalmente creadores en el ámbito científico, literario o artístico ban servido de argumento a los teóricos del proceso asociativo, por ello se bace una referencia a estos trabajos en la primera parte del artículo, para, posteriormente, centrar la revision en el análisis de la teoria asociativa de Mednick, como la más elaborada y sistemática de cuantas aportaciones se ban becho a la definición del pensamiento creador como un proceso asociativo. Se ofrecen datos del propio grupo de Mednick sobre argumentos empiricos de validación de la teoria y se plantean las limitaciones de la validez de la misma en relación con el instrumento de evaluación de la aptitud creadora en términos asociativos: el "Remote Associated Test».

\section{Summary}

This work is a revision of contributions to psychology of creativity proceeding from associative explanations of the creative process. The introspective reports of excepcionally creative individuals in scientific, artistic or literary fields have served as an argument for the theorist of associative process; due to it, I make a reference of such works in the first part of the article, centering, afterwards, the revision about the Mednick's associative theory as the most sistematic and treated of the ones made about the definition about creative thinking as an associative process.

Data are offered from Mednick's group about the empiric arguments in the validation of the theory; also restrictions of its validity are established in relation with the instrument gived for evaluation of creative ability in associative terms: the "Remote Associated Test 


\section{Résumé}

Cet article est une révision des apportations à la psychology de la creativité qui viennent des interpretations associatives du process creative.

Les references introspectives des subjects exceptionellement createurs dans le domain scientific, literaire ou artistic ont servi de sujet pour les theoriciens du process associative, donc, on fait reférence à ces trabaux dans la première part de mon article, pur àpres centrer la revision dans l'analyse de la theorie associative de Mednick, comme la plus elaborée et systematique des tous les apportations faites a la definition de la pensée creative comme un process associative.

On propose renseignements du propre proup de Mednick sur arguments empiriques de validation de la theorie et on present aussi les limitations de la validité de la même in relation avec l'instrument d'evaluation des aptitudes creatives en terms associatives: "The Remote Associated Testw.

Madrid, 29 de julio de 1983 